\title{
Localization and Universality of Eigenvectors in Directed Random Graphs
}

\author{
Fernando Lucas Metz $\odot$ \\ Physics Institute, Federal University of Rio Grande do Sul, 91501-970 Porto Alegre, Brazil \\ and London Mathematical Laboratory, 18 Margravine Gardens, London W6 8RH, United Kingdom \\ Izaak Neriఠ \\ Department of Mathematics, King's College London, Strand, London WC2R 2LS, United Kingdom
}

(Received 27 July 2020; revised 7 December 2020; accepted 8 January 2021; published 29 January 2021)

\begin{abstract}
Although the spectral properties of random graphs have been a long-standing focus of network theory, the properties of right eigenvectors of directed graphs have so far eluded an exact analytic treatment. We present a general theory for the statistics of the right eigenvector components in directed random graphs with a prescribed degree distribution and with randomly weighted links. We obtain exact analytic expressions for the inverse participation ratio and show that right eigenvectors of directed random graphs with a small average degree are localized. Remarkably, if the fourth moment of the degree distribution is finite, then the critical mean degree of the localization transition is independent of the degree fluctuations, which is different from localization in undirected graphs that is governed by degree fluctuations. We also show that in the high connectivity limit the distribution of the right eigenvector components is solely determined by the degree distribution. For delocalized eigenvectors, we recover in this limit the universal results from standard random matrix theory that are independent of the degree distribution, while for localized eigenvectors the eigenvector distribution depends on the degree distribution.
\end{abstract}

DOI: 10.1103/PhysRevLett.126.040604

Introduction.-Complex systems, such as neural networks [1-3], ecosystems [4], gene regulatory networks [5-7], social networks [8,9], and the World Wide Web $[10,11]$ are described by large, directed graphs. Therefore, there is much interest in understanding how the topology of directed graphs impacts the dynamics of processes and algorithms on them.

Much insight in the dynamical processes on graphs is gained from the spectral properties of the adjacency matrix that represents the network. This is because the linearized dynamics of a complex system in the vicinity of a fixed point is determined by the spectral properties of the adjacency matrix [12,13]. As a consequence, spectral analysis of the adjacency matrix has proven to be important in the study of neural networks [14-18], ecosystems [19-21], gene regulatory networks [22,23], and disease spreading [24-28]. In these systems, the eigenvectors of the adjacency matrix determine the dynamical modes evoked by external perturbations. In addition, right eigenvectors of adjacency matrices of directed graphs are used in algorithms for node centrality [29-31], community detection [32-34], and matrix completion [35].

In disordered systems, eigenvectors localize when the strength of the disorder is large enough [36,37]. Localized eigenvectors occupy a few vertices, whereas delocalized eigenvectors are extended over the whole system. The transition from a delocalized to a localized state leads to a qualitative change in the dynamics of processes and algorithms. For example, the localization transition implies a metal-insulator phase transition in solid state physics [36,37], a transition from an algorithmic successful to a failure phase in spectral algorithms [31,35,38], and a transition from a regime where the linear dynamics of a large complex system is governed by a finite number of vertices to a regime where the dynamics is governed by a finite fraction of all vertices. In the context of disease spreading, eigenvector localization implies that the fraction of infected vertices is very small right above the epidemic threshold [25].

For undirected random graphs, the localization of eigenvectors of the adjacency matrix has been well studied $[25,26,36,37,39-49]$. The eigenvector of the largest eigenvalue is localized if the maximal degree of the graph is larger than a certain value. Hence, degree fluctuations are crucial for the localization of eigenvectors in undirected graphs.

For directed random graphs, the statistical properties and the localization of eigenvectors have been studied for one-dimensional chains, such as the Hatano-Nelson model [50-52] and its extensions to biological systems [53,54], and a diluted Ginibre ensemble [55]. However, the localization of eigenvectors in directed random graphs that model complex systems, such as the World Wide Web or neural networks, have not been studied so far.

In this Letter, we make a significant step forward by developing an exact theory for the statistical properties of the right (or left) eigenvectors of directed random graphs 
with a prescribed degree distribution and random couplings. We derive exact analytic expressions for the inverse participation ratio and for the critical point of the localization-delocalization transition. Surprisingly, when the moments of the degree distribution are finite, the critical point of the localization-delocalization transition is independent of the degree distribution. Moreover, the right eigenvectors are localized if the degree distribution has a diverging fourth moment. We also show that in the high connectivity limit the statistics of the components of right eigenvectors are only determined by the degree distribution. In this limit, we obtain distinct universality classes that depend on an exponent that quantifies the degree fluctuations.

Model setup.-We consider random matrices $\mathbf{A}$ of dimension $n \times n$ with elements

$$
A_{i j}=J_{i j} C_{i j}, \quad i, j \in\{1,2, \ldots, n\},
$$

where $C_{i j} \in\{0,1\}$ are the entries of the adjacency matrix $\boldsymbol{C}$ of a simple and directed random graph with a prescribed degree distribution

$$
p_{K^{\text {in }}, K^{\text {out }}}(k, \ell)=p_{K^{\text {in }}}(k) p_{K^{\text {out }}}(\ell)
$$

of in degrees $K^{\text {in }}$ and out degrees $K^{\text {out }}$. We set $C_{i j}=1$ when there exists a directed link pointing from $i$ to $j$, such that the out degree (in degree) of the $i$ th node is $K_{i}^{\text {out }}=\sum_{j=1}^{n} C_{i j}\left(K_{i}^{\text {in }}=\sum_{j=1}^{n} C_{j i}\right)$. The $J_{i j}$ are real valued, independent, and identically distributed random variables drawn from a distribution $p_{J}(x)$.

Random graph models with undirected edges and a prescribed degree distribution are surveyed in Ref. [56]. Here we consider their extension to the directed case. Directed random graphs with a prescribed degree distribution [57-62] model the World Wide Web [10,11] and neural networks $[1,3,63]$. In this model, the in degrees and out degrees are drawn from Eq. (2) subject to the constraint $\sum_{j=1}^{n} K_{j}^{\text {in }}=\sum_{j=1}^{n} K_{j}^{\text {out }}$, and subsequently nodes are randomly connected according to the given degree sequences. Hence, given a sequence of degrees, random graphs are drawn uniformly from the set of simple and directed graphs. This model provides the ideal setting to explore the influence of network topology on the spectral properties of $\boldsymbol{A}$.

In what follows, brackets $\langle\cdot\rangle$ denote the average with respect to the distribution of $\boldsymbol{A}$. In particular, we use

$$
c=\left\langle K^{\text {out }}\right\rangle
$$

for the mean out degree, and we denote the variance of a random variable $X$ by $\operatorname{var}(X)=\left\langle X^{2}\right\rangle-\langle X\rangle^{2}$.

Spectra of infinitely large matrices $\mathbf{A}$. - The spectrum of A has been studied in Refs. [64-67]. For $n \rightarrow \infty$ and $c>1$, directed random graphs have a giant strongly connected component [68] and the spectral distribution $\rho_{\mathbf{A}}(\lambda)=$ $n^{-1} \sum_{j=1}^{n} \delta\left[\lambda-\lambda_{j}(\mathbf{A})\right]$ of the eigenvalues $\left\{\lambda_{j}(\mathbf{A})\right\}_{j=1}^{n}$ is

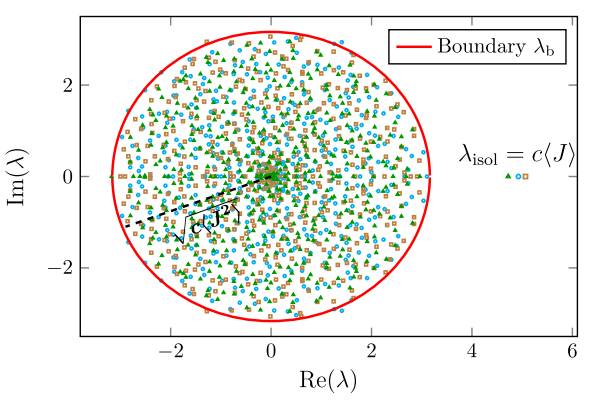

FIG. 1. Eigenvalues of three realizations (circles, triangles, and squares) of the adjacency matrix $\boldsymbol{A}$ of directed random graphs with $n=500$ [see Eq. (1)]. The in degrees and out degrees follow a Poisson distribution with average $c=5$. The weights $J_{i j}$ are drawn from a Gaussian distribution $p_{J}$ with mean and variance equal to 1 .

supported on a disk of radius $\left|\lambda_{\mathrm{b}}\right|=\sqrt{c\left\langle J^{2}\right\rangle}$ centered at the origin of the complex plane. In addition, if

$$
c>c_{\text {gap }}=\frac{\left\langle J^{2}\right\rangle}{\langle J\rangle^{2}},
$$

then there exists an eigenvalue outlier located at $\lambda_{\text {isol }}=c\langle J\rangle$ that is separated from the boundary $\lambda_{b}$ by a finite gap. Figure 1 shows the eigenvalues for an example of a directed random graph, where one clearly identifies the outlier $\lambda_{\text {isol }}$ and the boundary $\lambda_{b}$ of $\rho_{\mathbf{A}}(\lambda)$ for $n \rightarrow \infty$.

Distribution of the right eigenvector components. $-\mathrm{A}$ right eigenvector $\vec{R}(\lambda)$ associated to an eigenvalue $\lambda$ of $\boldsymbol{A}$ satisfies

$$
A \vec{R}(\lambda)=\lambda \vec{R}(\lambda)
$$

and the distribution of the entries of $\vec{R}(\lambda)$ reads

$$
p_{R}(r \mid \lambda)=\lim _{n \rightarrow \infty} \frac{1}{n} \sum_{i=1}^{n} \delta\left[r-R_{i}(\lambda)\right] .
$$

If $\lambda$ is an outlier ( $\left.\lambda=\lambda_{\text {isol }}\right)$ or $\lambda$ is located at the boundary of the spectrum $\left(\lambda=\lambda_{b}\right)$, then $p_{R}(r \mid \lambda)$ fulfills [65-67]

$$
\begin{aligned}
p_{R}(r \mid \lambda)= & \sum_{k=0}^{\infty} p_{K^{\text {out }}}(k) \int\left(\prod_{j=1}^{k} d x_{j} d^{2} r_{j} p_{J}\left(x_{j}\right) p_{R}\left(r_{j} \mid \lambda\right)\right) \\
& \times \delta\left(r-\frac{1}{\lambda} \sum_{j=1}^{k} x_{j} r_{j}\right),
\end{aligned}
$$

where $d^{2} r \equiv d \operatorname{Re} r d \operatorname{Im} r$. Equation (7) is exact for infinitely large and directed random graphs with a prescribed degree distribution, because they are locally treelike. In fact, the solutions of Eq. (7) are well corroborated by direct diagonalizations of large adjacency matrices [65-67]. The analytic results presented below follow from Eq. (7). 
Inverse participation ratio.-The localization of $\vec{R}(\lambda)$ can be characterized in terms of the inverse participation ratio (IPR) $[44,69,70]$

$$
\mathcal{I}(\lambda) \equiv \lim _{n \rightarrow \infty} \frac{n \sum_{i=1}^{n}\left|R_{i}(\lambda)\right|^{4}}{\left(\sum_{i=1}^{n}\left|R_{i}(\lambda)\right|^{2}\right)^{2}}=\frac{\left\langle|R(\lambda)|^{4}\right\rangle}{\left\langle|R(\lambda)|^{2}\right\rangle^{2}}
$$

where we have used that $\mathcal{I}$ is self-averaging [71]. The IPR is finite if $\vec{R}(\lambda)$ is delocalized, whereas $\mathcal{I}(\lambda)$ diverges if $\vec{R}(\lambda)$ is localized on a finite number of nodes.

From Eq. (7), we derive in the Supplemental Material [71] exact expressions for the IPR when $\lambda=\lambda_{\text {isol }}$ or $\lambda=\lambda_{b}$. We find that

$$
\mathcal{I}\left(\lambda_{b}\right)=\frac{(\gamma+1)\left[\left\langle\left(K^{\mathrm{out}}\right)^{2}\right\rangle-c\right]}{c\left(c-\left\langle J^{4}\right\rangle /\left\langle J^{2}\right\rangle^{2}\right)},
$$

where $\gamma=2$ when $\lambda_{b} \in \mathbb{R}$ and $\gamma=1$ when $\lambda_{b} \notin \mathbb{R}$. Analogously, the IPR at $\lambda=\lambda_{\text {isol }}$ reads

$$
\begin{aligned}
\mathcal{I}\left(\lambda_{\text {isol }}\right)= & \frac{3 \beta_{1}\left\langle J^{2}\right\rangle^{2}}{\left(c^{4}\langle J\rangle^{4}-c\left\langle J^{4}\right\rangle\right)}+\frac{\beta_{3}\left(c^{2}\langle J\rangle^{2}-c\left\langle J^{2}\right\rangle\right)^{2}}{\beta_{1}^{2}\left(c^{4}\langle J\rangle^{4}-c\left\langle J^{4}\right\rangle\right)} \\
& +\frac{12 \beta_{1}\left\langle J^{3}\right\rangle\left\langle J^{2}\right\rangle\left(c^{2}\langle J\rangle^{2}-c\left\langle J^{2}\right\rangle\right)}{\left(c^{4}\langle J\rangle^{4}-c\left\langle J^{4}\right\rangle\right)\left(c^{3}\langle J\rangle^{3}-c\left\langle J^{3}\right\rangle\right)} \\
& +\frac{4 \beta_{2}\left\langle J^{3}\right\rangle\left(c^{2}\langle J\rangle^{2}-c\left\langle J^{2}\right\rangle\right)^{2}}{\beta_{1}\left(c^{4}\langle J\rangle^{4}-c\left\langle J^{4}\right\rangle\right)\left(c^{3}\langle J\rangle^{3}-c\left\langle J^{3}\right\rangle\right)} \\
& +\frac{6 \beta_{2}\left\langle J^{2}\right\rangle\left(c^{2}\langle J\rangle^{2}-c\left\langle J^{2}\right\rangle\right)}{\beta_{1}\left(c^{4}\langle J\rangle^{4}-c\left\langle J^{4}\right\rangle\right)}
\end{aligned}
$$

where

$\beta_{\ell} \equiv \sum_{k=\ell+1}^{\infty} p_{K^{\text {out }}}(k) \frac{k !}{(k-\ell-1) !}, \quad \ell=1,2,3$.

Figure 2 illustrates Eqs. (9) and (10) as a function of $c$ for a Gaussian distribution $p_{J}$ and three different out degree distributions: Poisson, exponential, and Borel distribution (see Supplemental Material [71]). All moments of these degree distributions are finite and each $p_{K^{\text {out }}}$ is parametrized only by $c$. Figure 2 shows that the IPR is finite if $c$ is large enough and it diverges for small $c$, which demonstrates the existence of a delocalization-localization phase transition in directed random graphs.

The localization phase transition.-There are two mechanisms for localization, one governed by fluctuations of $J_{i j}$, and a second one governed by degree fluctuations.

The first mechanism is illustrated in Fig. 2 and it holds for arbitrary $p_{K^{\text {out }}}$ with a finite fourth moment. In this case, the right eigenvectors associated to $\lambda=\lambda_{b}$ and $\lambda=\lambda_{\text {isol }}$ are localized when $c$ is smaller than

$$
c_{b}=\frac{\left\langle J^{4}\right\rangle}{\left\langle J^{2}\right\rangle^{2}} \quad \text { and } \quad c_{\text {isol }}^{3}=\frac{\left\langle J^{4}\right\rangle}{\langle J\rangle^{4}},
$$
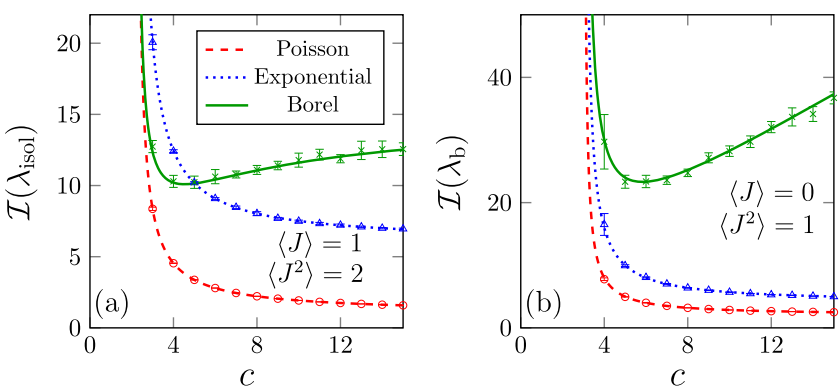

FIG. 2. The IPR $\mathcal{I}(\lambda)$ of right eigenvectors associated with $\lambda_{\text {isol }}$ [panel (a)] and $\lambda_{b} \notin \mathbb{R}$ [panel (b)]. Equations (9) and (10) (different line styles) are shown as a function of the average degree $c$ for different out degree distributions: Poisson, exponential, and Borel (see Supplemental Material [71]). The weights $J_{i j}$ are drawn from a Gaussian distribution $p_{J}$ with first and second moments indicated on each panel. The symbols are obtained from the numerical solutions of Eq. (7) using the population dynamics algorithm $[43,66]$, while direct diagonalization results for $\mathcal{I}(\lambda)$ are presented in the Supplemental Material [71]. The error bars are the standard deviation of the IPR for 10 independent runs of population dynamics. The results for the Borel distribution are rescaled as $\mathcal{I}\left(\lambda_{\text {isol }}\right) \rightarrow \mathcal{I}\left(\lambda_{\text {isol }}\right) / c$ in panel (a).

respectively. Thus, the critical points for the localization transitions only depend on the lower moments of $p_{J}$ and they are independent of $p_{K^{\text {out }}}$. When $p_{J}(x)=\delta(x-1)$, we obtain $c_{b}=c_{\text {isol }}=1$ and the delocalization-localization transition is governed by the percolation transition for the strongly connected component [68]. According to Eq. (10), a localization transition at $c_{\text {isol }}^{*}=\sqrt{\left\langle J^{3}\right\rangle /\langle J\rangle^{3}}$ is, in principle, possible, but we could not find an example of $p_{J}$ for which $c_{\text {isol }}^{*}>c_{\text {isol }}$ and $c_{\text {isol }}^{*}>c_{\text {gap }}$.

Figure 3 shows the phase diagram when $p_{J}$ is a Gaussian distribution with mean $\mu$ and variance $\sigma^{2}$. In this case, $c_{\text {gap }}$, $c_{b}$, and $c_{\text {isol }}$ only depend on $\sigma / \mu$. A few generic properties of eigenvector localization in directed random graphs, which also hold for non-Gaussian $p_{J}$, are illustrated in Fig. 3. First, $\vec{R}\left(\lambda_{\text {isol }}\right)$ is delocalized when $\left\langle J^{2}\right\rangle^{3}>\left\langle J^{4}\right\rangle\langle J\rangle^{2}$ because $c_{\text {gap }}>c_{\text {isol }}$. Second, the transition lines fulfill

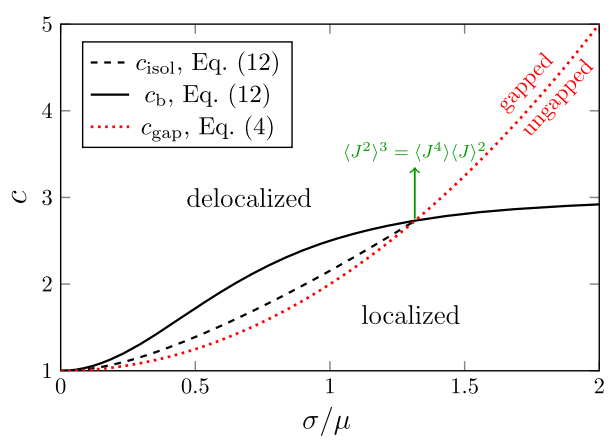

FIG. 3. Phase diagram for the localization of right eigenvectors associated to $\lambda_{\text {isol }}$ and $\lambda_{b}$. The distribution $p_{J}$ is Gaussian with mean $\mu$ and standard deviation $\sigma$. 
$c_{\text {gap }}<c_{\text {isol }}<c_{b}$ for $\left\langle J^{2}\right\rangle^{3}<\left\langle J^{4}\right\rangle\langle J\rangle^{2}$. Lastly, the critical transitions $c_{\text {gap }}, c_{\text {isol }}$, and $c_{\text {gap }}$ intersect in a common point because $c_{\text {isol }}^{3}=c_{b} c_{\text {gap }}^{2}$.

The second mechanism for localization is due to large degree fluctuations. From Eqs. (9) and (10), it follows that $\mathcal{I}\left(\lambda_{b}\right) \rightarrow \infty$ if $\left\langle\left(K^{\text {out }}\right)^{2}\right\rangle \rightarrow \infty$ and $\mathcal{I}\left(\lambda_{\text {isol }}\right) \rightarrow \infty$ if $\left\langle\left(K^{\text {out }}\right)^{4}\right\rangle \rightarrow \infty$, independently of $p_{J}$. Hence, localization of $\vec{R}\left(\lambda_{b}\right)$ and $\vec{R}\left(\lambda_{\text {isol }}\right)$ also occurs in graphs with power-law degree distributions. In the sequel, we show that degreebased localization persists in the high connectivity limit.

Localization and universality in the high connectivity limit.-In Fig. 2, $\mathcal{I}(\lambda)$ flows to different asymptotic values for $c \gg 1$. To explore the localization and universality of eigenvectors in the high connectivity limit $c \rightarrow \infty$, we analyze the moments of the distribution $p_{R}$. Since $\left\langle R\left(\lambda_{\text {isol }}\right)\right\rangle$ is finite, we characterize the limit $c \rightarrow \infty$ of $p_{R}\left(r \mid \lambda_{\text {isol }}\right)$ through the relative variance

$$
\mathcal{R}_{c}=\frac{\operatorname{var}\left[R\left(\lambda_{\text {isol }}\right)\right]}{\left\langle R\left(\lambda_{\text {isol }}\right)\right\rangle^{2}} .
$$

On the other hand, since $\left\langle R\left(\lambda_{b}\right)\right\rangle=0$, we characterize the limit $c \rightarrow \infty$ of $p_{R}\left(r \mid \lambda_{b}\right)$ through the kurtosis

$$
\mathcal{K}_{c}=\frac{\left\langle\left(\operatorname{Re} R\left(\lambda_{b}\right)\right)^{4}\right\rangle}{\left\langle\left(\operatorname{Re} R\left(\lambda_{b}\right)\right)^{2}\right\rangle^{2}}=\frac{(4-\gamma)}{2} \mathcal{I}\left(\lambda_{b}\right),
$$

where we used the fact that odd moments of $p_{R}\left(r \mid \lambda_{b}\right)$ are zero [71]. Setting $c \rightarrow \infty$ in Eqs. (13) and (14), we obtain [71]

$$
\begin{gathered}
\mathcal{R}_{\infty}=\lim _{c \rightarrow \infty} \frac{\operatorname{var}\left[K^{\text {out }}\right]}{c^{2}}, \\
\mathcal{K}_{\infty}=3\left(1+\lim _{c \rightarrow \infty} \frac{\operatorname{var}\left[K^{\text {out }}\right]}{c^{2}}\right),
\end{gathered}
$$

which indicates that the limit $c \rightarrow \infty$ of $p_{R}$ is determined by the degree distribution. We see that, in general, $p_{R}\left(r \mid \lambda_{b}\right)$ and $p_{R}\left(r \mid \lambda_{\text {isol }}\right)$ are not Gaussian in the high connectivity limit.

With the purpose of classifying the universal behavior of $p_{R}$ for $c \rightarrow \infty$, let us consider degree distributions that satisfy

$$
\operatorname{var}\left[K^{\text {out }}\right]=B c^{\alpha}(c \gg 1),
$$

where $\alpha$ and $B$ depend on the specific choice of $p_{K^{\text {out }}}(k)$. Equation (17) holds for different examples of degree distributions, including those in Fig. 2. Plugging this ansatz for $\operatorname{var}\left[K^{\text {out }}\right]$ in Eqs. (15) and (16), we obtain three universality classes for $\lim _{c \rightarrow \infty} p_{R}(r \mid \lambda)$, which are determined by the exponent $\alpha$ that controls the degree fluctuations. The results for the universality classes are summarized in Table I. In terms of $\mathcal{R}_{\infty}$ and $\mathcal{K}_{\infty}$, we find that for $\alpha \leq 2$ the
TABLE I. The relative variance $\mathcal{R}_{c}$ of $\vec{R}\left(\lambda_{\text {isol }}\right)$ and the kurtosis $\mathcal{K}_{c}$ of $\vec{R}\left(\lambda_{b}\right)$ in the high connectivity limit $c \rightarrow \infty$ [see Eqs. (15) and (16)], together with an example of the out degree distribution $p_{K^{\text {out }}}$ in each regime of $\alpha$ [see Eq. (17)].

\begin{tabular}{lccc}
\hline \hline & $\alpha<2$ & $\alpha=2$ & $\alpha>2$ \\
\hline $\mathcal{R}_{\infty}$ & 0 & $B$ & $\infty$ \\
$\mathcal{K}_{\infty}$ & 3 & $3(1+B)$ & $\infty$ \\
Example & Poisson & Exponential & Borel \\
\hline \hline
\end{tabular}

eigenvectors $\vec{R}\left(\lambda_{b}\right)$ and $\vec{R}\left(\lambda_{\text {isol }}\right)$ are delocalized in the limit $c \rightarrow \infty$, whereas for $\alpha>2$ these eigenvectors are localized due to large degree fluctuations.

The eigenvector distributions in the high connectivity limit.-The results in Table I indicate that $p_{R}(r \mid \lambda)$ is universal for $c \rightarrow \infty$. Below we present explicit expressions for $p_{R}(r \mid \lambda)$ when $c \rightarrow \infty$. Henceforth we set $\left\langle|R|^{2}\right\rangle=1$ without losing generality.

The characteristic function of $p_{R}(r \mid \lambda)$ is given by [71]

$$
g_{R}(u, v \mid \lambda)=\sum_{k=0}^{\infty} p_{K^{\text {out }}}(k) e^{k \ln F(u, v \mid \lambda)},
$$

where

$F(u, v \mid \lambda)=\int d x p_{J}(x) \int d^{2} r p_{R}(r \mid \lambda) \exp ^{-\frac{x z r}{2 \lambda}+\frac{x z^{*} r^{*}}{2 \lambda^{*}}}$,

and $z=u+i v$. The symbol $(\ldots)^{*}$ denotes complex conjugation. If $\lambda \in \mathbb{R}$, the eigenvector components are real and $F(u, v \mid \lambda)$ is independent of $v$.

Setting $\lambda=\lambda_{\text {isol }}$ or $\lambda=\lambda_{b}$ in Eq. (19), we can expand $F(u, v \mid \lambda)$ for $c \gg 1$ up to order $O(1 / c)$ if $\alpha \leq 2$ (see Table I). This approach does not work for $\alpha>2$, because the moments of $p_{R}$ can diverge in this regime. Thus, performing this expansion for $\alpha \leq 2$ and substituting the resulting expression for $F(u, v \mid \lambda)$ in Eq. (18), we obtain [71]

$g_{R}\left(u, v \mid \lambda_{b}\right)=\sum_{k=0}^{\infty} p_{K^{\text {out }}}(k) \exp \left[-\frac{\gamma k}{4 c}\left(u^{2}+(2-\gamma) v^{2}\right)\right]$,

$g_{R}\left(u, v \mid \lambda_{\text {isol }}\right)=\sum_{k=0}^{\infty} p_{K^{\text {out }}}(k) \exp \left(-\frac{i u k}{c \sqrt{B c^{\alpha-2}+1}}\right)$.

Remarkably, the characteristic functions for $c \rightarrow \infty$ are fully specified by $p_{K^{\text {out }}}$ and they are independent of $p_{J}$.

For degree distributions where $\lim _{c \rightarrow \infty} \operatorname{var}\left[K^{\text {out }}\right] / c^{2}=0$ $(\alpha<2)$, we can set $p_{K^{\text {out }}}(k)=\delta_{k, c}$ in Eqs. (20) and (21), leading to [71]

$$
p_{R}\left(r \mid \lambda_{b}\right)=\frac{1}{\pi} e^{-|r|^{2}}\left(\lambda_{b} \notin \mathbb{R}\right)
$$



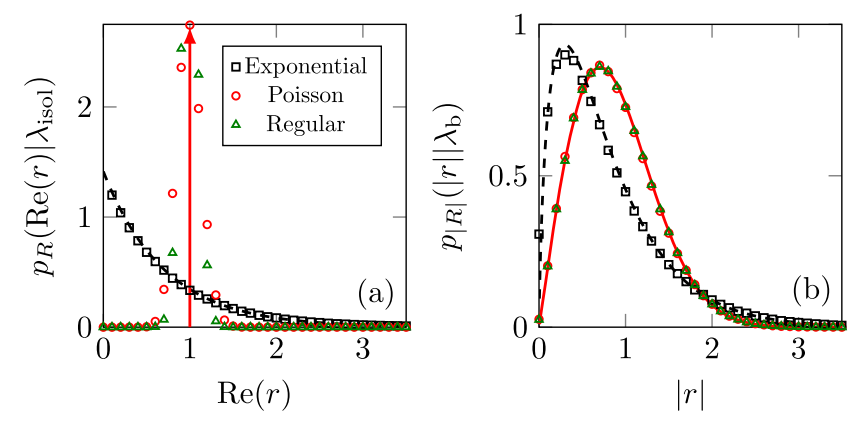

FIG. 4. The high connectivity limit $c \rightarrow \infty$ of the distribution $p_{R}\left[\operatorname{Re}(r) \mid \lambda_{\text {isol }}\right]$ of the real part of the eigenvector components at $\lambda_{\text {isol }}$ [panel (a)], and of the distribution $p_{|R|}\left(|r| \mid \lambda_{b}\right)$ of the norm of the eigenvector components at $\lambda_{b} \notin \mathbb{R}$ [panel (b)]. The solid red lines and the dashed black lines are, respectively, the analytic results for regular or Poisson and exponential degree distributions [see Eqs. (22)-(25)], while the symbols are numerical solutions of Eq. (7) with $c=100$. The numerical data for regular or Poisson graphs in panel (a) is a Gaussian distribution with variance of $O(1 / c)$, approaching the Dirac delta distribution (vertical arrow) for $c \rightarrow \infty$.

$$
p_{R}\left(r \mid \lambda_{\text {isol }}\right)=\delta[\operatorname{Im}(r)] \delta[\operatorname{Re}(r)-1]
$$

Equation (22) yields the well-known Porter-Thomas distribution for the eigenvector components of Gaussian random matrices [77,78]. Thus, standard results from random matrix theory are recovered when $\alpha<2$.

If $p_{K^{\text {out }}}$ is an exponential distribution, where $\alpha=2$, we obtain in the limit $c \rightarrow \infty$ [71]

$$
\begin{gathered}
p_{R}\left(r \mid \lambda_{b}\right)=\frac{2}{\pi} K_{0}(2|r|) \quad\left(\lambda_{b} \notin \mathbb{R}\right), \\
p_{R}\left(r \mid \lambda_{\text {isol }}\right)=\sqrt{2} \delta[\operatorname{Im}(r)] \Theta[\operatorname{Re}(r)] e^{-\sqrt{2} \operatorname{Re}(r),}
\end{gathered}
$$

where $\Theta(x)$ is the Heaviside step function and $K_{0}(x)$ is a modified Bessel function of the second kind [79]. Figure 4 illustrates the shape of the distributions $p_{R}$ given by Eqs. (22)-(25), and compares them with numerical solutions of Eq. (7) for $c=100$. The derivation of Eqs. (22)-(25) is explained in the Supplemental Material [71].

Conclusions. - We have shed light on the relationship between graph topology and the localization of right eigenvectors in directed random graphs. If the moments of the out degree distribution $p_{K^{\text {out }}}$ are finite, then right eigenvectors at the edge of the spectrum are localized below a critical mean out degree. It is striking that the critical points for the localization transitions are universal, in the sense they only depend on the lower moments of the distribution $p_{J}$ of the edge weights, regardless of the network topology. Therefore, localization in directed random graphs is fundamentally different from localization in undirected graphs, for which degree fluctuations are important [25,42-44,46,47,80,81]. Indeed, the eigenvector associated with the largest eigenvalue of the adjacency matrix of an undirected random graph is localized if the maximal degree is large enough [25]. Degree-based localization is also possible for directed random graphs, but then $p_{K^{\text {out }}}$ requires a divergent fourth moment.

In the high connectivity limit, the distribution $p_{R}$ of the right eigenvector components is determined by the graph topology, independently of $p_{J}$. If the out degree fluctuations are small enough, then eigenvectors are delocalized and $p_{R}$ is given by the same universal distribution as in the case of Gaussian random matrices $[77,78]$. On the other hand, if the out degree fluctuations are large enough, then eigenvectors are localized and the distribution $p_{R}$ depends on $p_{K^{\text {out }}}$. More generally, these results indicate that Gaussian random matrix theory describes well the spectral properties of high connectivity graphs only when the degree fluctuations are sufficiently small [82].

For future work, it would be interesting to explore the implications of eigenvector localization for the dynamics of neural networks [53,54] and ecosystems $[20,83]$, to compare the theoretical predictions for the IPR with empirical values in real-world networks [26,84], and to study eigenvector localization of Laplacians of directed graphs [85-87].

The authors thank Jacopo Grilli for interesting discussions. F. L. M. thanks the London Mathematical Laboratory and $\mathrm{CNPq} / \mathrm{Brazil}$ for financial support.

[1] N. Brunel, Dynamics of sparsely connected networks of excitatory and inhibitory spiking neurons, J. Comput. Neurosci. 8, 183 (2000).

[2] E. Bullmore and O. Sporns, Complex brain networks: Graph theoretical analysis of structural and functional systems, Nat. Rev. Neurosci. 10, 186 (2009).

[3] O. Sporns, Networks of the Brain (MIT Press, Cambridge, MA, 2010).

[4] J. Bascompte, Disentangling the web of life, Science $\mathbf{3 2 5}$, 416 (2009).

[5] R. Milo, S. Shen-Orr, S. Itzkovitz, N. Kashtan, D. Chklovskii, and U. Alon, Network motifs: Simple building blocks of complex networks, Science 298, 824 (2002).

[6] S. S. Shen-Orr, R. Milo, S. Mangan, and U. Alon, Network motifs in the transcriptional regulation network of escherichia coli, Nat. Genet. 31, 64 (2002).

[7] T. I. Lee et al., Transcriptional regulatory networks in saccharomyces Cerevisiae, Science 298, 799 (2002).

[8] H. Kwak, C. Lee, H. Park, and S. Moon, What is twitter, a social network or a news media?, in Proceedings of the 19th International Conference on World Wide Web (2010), pp. 591-600.

[9] L. M. Aiello, A. Barrat, R. Schifanella, C. Cattuto, B. Markines, and F. Menczer, Friendship prediction and homophily in social media, ACM Trans. Web (TWEB) 6, 1 (2012). 
[10] A. Broder, R. Kumar, F. Maghoul, P. Raghavan, S. Rajagopalan, R. Stata, A. Tomkins, and J. Wiener, Graph structure in the web, Comput. Netw. 33, 309 (2000).

[11] R. Pastor-Satorras and A. Vespignani, Evolution and Structure of the Internet: A Statistical Physics Approach (Cambridge University Press, Cambridge, England, 2007).

[12] P. Hartman, A lemma in the theory of structural stability of differential equations, Proc. Am. Math. Soc. 11, 610 (1960).

[13] D. M. Grobman, Homeomorphism of systems of differential equations, Dokl. Akad. Nauk SSSR 128, 880 (1959).

[14] H. Sompolinsky, A. Crisanti, and H.-J. Sommers, Chaos in Random Neural Networks, Phys. Rev. Lett. 61, 259 (1988).

[15] L. C. G. Del Molino, K. Pakdaman, J. Touboul, and G. Wainrib, Synchronization in random balanced networks, Phys. Rev. E 88, 042824 (2013).

[16] J. Kadmon and H. Sompolinsky, Transition to Chaos in Random Neuronal Networks, Phys. Rev. X 5, 041030 (2015).

[17] J. Aljadeff, M. Stern, and T. Sharpee, Transition to Chaos in Random Networks with Cell-Type-Specific Connectivity, Phys. Rev. Lett. 114, 088101 (2015).

[18] D. Martí, N. Brunel, and S. Ostojic, Correlations between synapses in pairs of neurons slow down dynamics in randomly connected neural networks, Phys. Rev. E 97, 062314 (2018).

[19] R. M. May, Will a large complex system be stable?, Nature (London) 238, 413 (1972).

[20] S. Allesina, J. Grilli, G. Barabás, S. Tang, J. Aljadeff, and A. Maritan, Predicting the stability of large structured food webs, Nat. Commun. 6, 7842 (2015).

[21] T. Gibbs, J. Grilli, T. Rogers, and S. Allesina, Effect of population abundances on the stability of large random ecosystems, Phys. Rev. E 98, 022410 (2018).

[22] Y. Chen, Y. Shen, P. Lin, D. Tong, Y. Zhao, S. Allesina, X. Shen, and C.-I. Wu, Gene regulatory network stabilized by pervasive weak repressions: microRNA functions revealed by the May-Wigner theory, Natl. Sci. Rev. 6, 1176 (2019).

[23] Y. Guo and A. Amir, Exploring the effect of network topology, mRNA and protein dynamics on gene regulatory network stability, Nat. Commun. 12, 130 (2021).

[24] P. Van Mieghem, Epidemic phase transition of the SIS type in networks, Europhys. Lett. 97, 48004 (2012).

[25] A. V. Goltsev, S. N. Dorogovtsev, J. G. Oliveira, and J. F. F. Mendes, Localization and Spreading of Diseases in Complex Networks, Phys. Rev. Lett. 109, 128702 (2012).

[26] R. Pastor-Satorras and C. Castellano, Eigenvector localization in real networks and its implications for epidemic spreading, J. Stat. Phys. 173, 1110 (2018).

[27] C. Li, H. Wang, and P. Van Mieghem, Epidemic threshold in directed networks, Phys. Rev. E 88, 062802 (2013).

[28] D. H. Silva and S. C. Ferreira, Dissecting localization phenomena of dynamical processes on networks, arXiv:2011.10918.

[29] P. Bonacich, Factoring and weighting approaches to status scores and clique identification, J. Math. Sociol. 2, 113 (1972).

[30] J. G. Restrepo, E. Ott, and B. R. Hunt, Characterizing the Dynamical Importance of Network Nodes and Links, Phys. Rev. Lett. 97, 094102 (2006).
[31] T. Martin, X. Zhang, and M. E. J. Newman, Localization and centrality in networks, Phys. Rev. E 90, 052808 (2014).

[32] F. Krzakala, C. Moore, E. Mossel, J. Neeman, A. Sly, L. Zdeborová, and P. Zhang, Spectral redemption in clustering sparse networks, Proc. Natl. Acad. Sci. U.S.A. 110, 20935 (2013).

[33] C. Bordenave, M. Lelarge, and L. Massoulié, Nonbacktracking spectrum of random graphs: Community detection and non-regular Ramanujan graphs, in 2015 IEEE 56th Annual Symposium on Foundations of Computer Science (IEEE, New York, 2015), pp. 1347-1357.

[34] T. Kawamoto, Algorithmic detectability threshold of the stochastic block model, Phys. Rev. E 97, 032301 (2018).

[35] C. Bordenave, S. Coste, and R. Rao Nadakuditi, Detection thresholds in very sparse matrix completion, arXiv:2005.06062.

[36] R. Abou-Chacra, D. J. Thouless, and P. W. Anderson, A selfconsistent theory of localization, J. Phys. C 6, 1734 (1973).

[37] M. Aizenman and S. Warzel, Extended States in a Lifshitz Tail Regime for Random Schrödinger Operators on trees, Phys. Rev. Lett. 106, 136804 (2011).

[38] O. Giraud, B. Georgeot, and D. L. Shepelyansky, Delocalization transition for the google matrix, Phys. Rev. E 80, 026107 (2009).

[39] Y. V. Fyodorov and A. D. Mirlin, Localization in Ensemble of Sparse Random Matrices, Phys. Rev. Lett. 67, 2049 (1991).

[40] A. D. Mirlin and Y V. Fyodorov, Localization transition in the anderson model on the Bethe lattice: Spontaneous symmetry breaking and correlation functions, Nucl. Phys. B366, 507 (1991).

[41] S. N. Evangelou, A numerical study of sparse random matrices, J. Stat. Phys. 69, 361 (1992).

[42] M. Bauer and O. Golinelli, Random incidence matrices: Moments of the spectral density, J. Stat. Phys. 103, 301 (2001).

[43] R. Kühn, Spectra of sparse random matrices, J. Phys. A 41, 295002 (2008).

[44] F. L. Metz, I. Neri, and D. Bollé, Localization transition in symmetric random matrices, Phys. Rev. E 82, 031135 (2010).

[45] Y. Kabashima and H. Takahashi, First eigenvalue/ eigenvector in sparse random symmetric matrices: Influences of degree fluctuation, J. Phys. A 45, 325001 (2012).

[46] F. Slanina, Localization of eigenvectors in random graphs, Eur. Phys. J. B 85, 361 (2012).

[47] R. Pastor-Satorras and C. Castellano, Distinct types of eigenvector localization in networks, Sci. Rep. 6, 18847 (2016).

[48] K. S. Tikhonov, A. D. Mirlin, and M. A. Skvortsov, Anderson localization and ergodicity on random regular graphs, Phys. Rev. B 94, 220203(R) (2016).

[49] V. A. R. Susca, P. Vivo, and R. Kühn, Top eigenpair statistics for weighted sparse graphs, J. Phys. A 52, 485002 (2019).

[50] N. Hatano and D. R. Nelson, Localization Transitions in Non-Hermitian Quantum Mechanics, Phys. Rev. Lett. 77, 570 (1996). 
[51] N. Hatano and D. R. Nelson, Vortex pinning and nonHermitian quantum mechanics, Phys. Rev. B 56, 8651 (1997).

[52] J. Feinberg and A. Zee, Non-Hermitian localization and delocalization, Phys. Rev. E 59, 6433 (1999).

[53] A. Amir, N. Hatano, and D. R. Nelson, Non-Hermitian localization in biological networks, Phys. Rev. E 93, 042310 (2016).

[54] G. H. Zhang and D. R. Nelson, Eigenvalue repulsion and eigenvector localization in sparse non-Hermitian random matrices, Phys. Rev. E 100, 052315 (2019).

[55] T. Peron, B. M. F. de Resende, F. A. Rodrigues, L. F. Costa, and J. A. Méndez-Bermúdez, Spacing ratio characterization of the spectra of directed random networks, Phys. Rev. E 102, 062305 (2020).

[56] B. K. Fosdick, D. B. Larremore, J. Nishimura, and J. Ugander, Configuring random graph models with fixed degree sequences, SIAM Rev. 60, 315 (2018),

[57] M. Molloy and B. Reed, A critical point for random graphs with a given degree sequence, Random Struct. Algorithms 6, 161 (1995).

[58] M. Molloy and B. Reed, The size of the giant component of a random graph with a given degree sequence, Combinatorics Probab. Comput. 7, 295 (1998).

[59] B. Bollobás and B. Béla, Random Graphs (Cambridge University Press, Cambridge, England, 2001), Vol. 73.

[60] M. E. J. Newman, S. H. Strogatz, and D. J. Watts, Random graphs with arbitrary degree distributions and their applications, Phys. Rev. E 64, 026118 (2001).

[61] M. Newman, Networks: An Introduction (OUP, Oxford, 2010).

[62] S. N. Dorogovtsev and J.F.F. Mendes, Evolution of Networks: From Biological Nets to the Internet and WWW (OUP, Oxford, 2013).

[63] The Handbook of Brain Theory and Neural Networks, edited by M. A. Arbib (MIT Press, Cambridge, MA, 2003).

[64] T. Rogers and I. P. Castillo, Cavity approach to the spectral density of non-Hermitian sparse matrices, Phys. Rev. E 79, 012101 (2009).

[65] I. Neri and F.L. Metz, Eigenvalue Outliers of NonHermitian Random Matrices with a Local Tree Structure, Phys. Rev. Lett. 117, 224101 (2016).

[66] F. L. Metz, I. Neri, and T. Rogers, Spectral theory of sparse non-Hermitian random matrices, J. Phys. A 52, 434003 (2019).

[67] I. Neri and F. L. Metz, Linear stability analysis of large dynamical systems on random directed graphs, Phys. Rev. Research 2, 033313 (2020).

[68] S. N. Dorogovtsev, J. F. F. Mendes, and A. N. Samukhin, Giant strongly connected component of directed networks, Phys. Rev. E 64, 025101(R) (2001).
[69] Y. V. Fyodorov and A. D. Mirlin, Statistical properties of eigenfunctions of random quasi 1d one-particle Hamiltonians, Int. J. Mod. Phys. B 08, 3795 (1994).

[70] K. Efetov, Supersymmetry in Disorder and Chaos (Cambridge University Press, Cambridge, England, 1999).

[71] See Supplemental Material at http://link.aps.org/ supplemental/10.1103/PhysRevLett.126.040604 for all derivations, which also includes Refs. [72-76].

[72] A. Dembo and A. Montanari, Gibbs measures and phase transitions on sparse random graphs, Braz. J. Probab. Stat. 24, 137 (2010).

[73] B. Bollobás, A probabilistic proof of an asymptotic formula for the number of labelled regular graphs, Eur. J. Combinatorics 1, 311 (1980).

[74] P. van Mieghem, Graph Spectra for Complex Networks (Cambridge University Press, Cambridge, England, 2012).

[75] E. Borel, Sur l'emploi du théorème de Bernoulli pour faciliter le calcul d'une infinité de coefficients. Application au problème de l'attente à un guichet, C. R. Acad. Sci. 214, 452 (1942).

[76] J.C. Tanner, A derivation of the Borel distribution, Biometrika 48, 222 (1961).

[77] C. E. Porter and R. G. Thomas, Fluctuations of nuclear reaction widths, Phys. Rev. 104, 483 (1956).

[78] A. D. Mirlin, Statistics of energy levels and eigenfunctions in disordered systems, Phys. Rep. 326, 259 (2000).

[79] I. S. Gradshteyn and I. M. Ryzhik, Table of Integrals, Series, and Products (Elsevier Science, New York, 2014).

[80] M. Krivelevich and B. Sudakov, The largest eigenvalue of sparse random graphs, Combinatorics Probab. Comput. 12, 61 (2003).

[81] R. R. Nadakuditi and M. E. J. Newman, Spectra of random graphs with arbitrary expected degrees, Phys. Rev. E 87, 012803 (2013).

[82] F. L. Metz and J. D. Silva, Spectral density of dense random networks and the breakdown of the Wigner semicircle law, Phys. Rev. Research 2, 043116 (2020).

[83] J. Grilli, T. Rogers, and S. Allesina, Modularity and stability in ecological communities, Nat. Commun. 7, 12031 (2016).

[84] S. N. Dorogovtsev, A. V. Goltsev, J. F. F. Mendes, and A. N. Samukhin, Spectra of complex networks, Phys. Rev. E 68, 046109 (2003).

[85] J. Stäring, B. Mehlig, Y. V. Fyodorov, and J. M. Luck, Random symmetric matrices with a constraint: The spectral density of random impedance networks, Phys. Rev. E 67, 047101 (2003).

[86] A. N. Samukhin, S. N. Dorogovtsev, and J. F. F. Mendes, Laplacian spectra of, and random walks on, complex networks: Are scale-free architectures really important?, Phys. Rev. E 77, 036115 (2008).

[87] R. Kühn, Spectra of random stochastic matrices and relaxation in complex systems, Europhys. Lett. 109, 60003 (2015). 